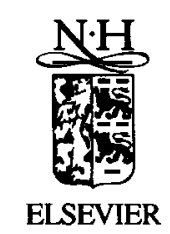

Journal of Luminescence 58 (1994) 413-416

\title{
Nonclassical crossovers in binary reactions in one-dimensional systems
}

\author{
Panos Argyrakis ${ }^{\mathrm{a}, *}$, Raoul Kopelman ${ }^{\mathrm{a}}$, Katja Lindenberg ${ }^{\mathrm{b}}$ \\ ${ }^{2}$ Departments of Chemistry and Physics, University of Michigan, Ann Arbor, MI 48109-1055, USA \\ ${ }^{\mathrm{b}}$ Department of Chemistry and Institute for Nonlinear Science, University of California at San Diego, La Jolla, CA 92093-0340, USA
}

\begin{abstract}
We examine the crossover behavior for the $\mathrm{A}+\mathrm{A} \rightarrow 0$ and $\mathrm{A}+\mathrm{B} \rightarrow 0$ reactions, which are highly nonclassical for a random initial distribution of reactants. We find that both reactions quickly depart from the classical behavior to a "depletion zone regime", eventually reaching the segregated asymptotic behavior. We give the corresponding scaling laws for the crossover time and density. Finite size effects are also discussed. Computer simulations verify the scaling laws and provide the scaling coefficients.
\end{abstract}

Chemical reactions in low dimensions that are diffusion controlled do not behave classically but exhibit "anomalous" rate laws for the densities $\rho(t)$ of the reacting species [1-8]. Thus, for the model reaction $\mathrm{A}+\mathrm{A} \rightarrow 0$ while the normal rate law is $\dot{\rho}=-k \rho^{2}$, for dimensions $d<2$ it is $\dot{\rho}=$ $-k \rho^{(1+2 / d)}[1,4,8]$. It is now well understood that this behavior is a consequence of the spatial distribution of A's: Initially the distribution has a maximum at zero separation, but as the reaction proceeds the particles that are in close proximity have the chance to react, thus leaving a depletion zone and a distribution that peaks at a finite, nonzero nearest neighbor separation. A similar situation holds for the reaction $\mathrm{A}+\mathrm{B} \rightarrow 0$. Under normal

\footnotetext{
* Corresponding author. Permanent address: Department of Physics, University of Thessaloniki, GR-54006 Thessaloniki, Greece.
}

circumstances the rate laws are $\dot{\rho}_{\mathrm{A}}=\dot{\rho}_{\mathrm{B}}=$ $-k \rho_{\mathrm{A}} \rho_{\mathrm{B}}$. If $\rho_{\mathrm{A}}(t=0)=\rho_{\mathrm{B}}(t=0) \equiv \rho(t=0)$, then the densities of the two species are equal, so $\dot{\rho}=-k \rho^{2}$. But, again it is well known that the actual rate law in dimensions $d<4$ is instead $\dot{\rho}=-k \rho^{(1+4 / d)}$. Here the cause of the anomalous behavior is the formation of aggregates of like particles [1-8]. The regions where the density of one type of particles is overwhelmingly greater than that of the other grow in time. Since the reaction can essentially occur only at the interfaces between aggregates, and since the number of these interfaces decreases with time, the reaction slows down relative to the rate that would describe a random mixture of reactants. Again, this behavior reflects the fact that diffusion is not an effective mixing mechanism in low dimensions. Initial spatial fluctuations in relative densities can thus grow in size as the reaction that eliminates close opposite pairs proceeds. In this paper we briefly discuss the various regimes of kinetic behavior from the initial time

0022-2313/94/\$07.00 (C) 1994 - Elsevier Science B.V. All rights reserved SSDI 0022-2313(93) E0218-M 


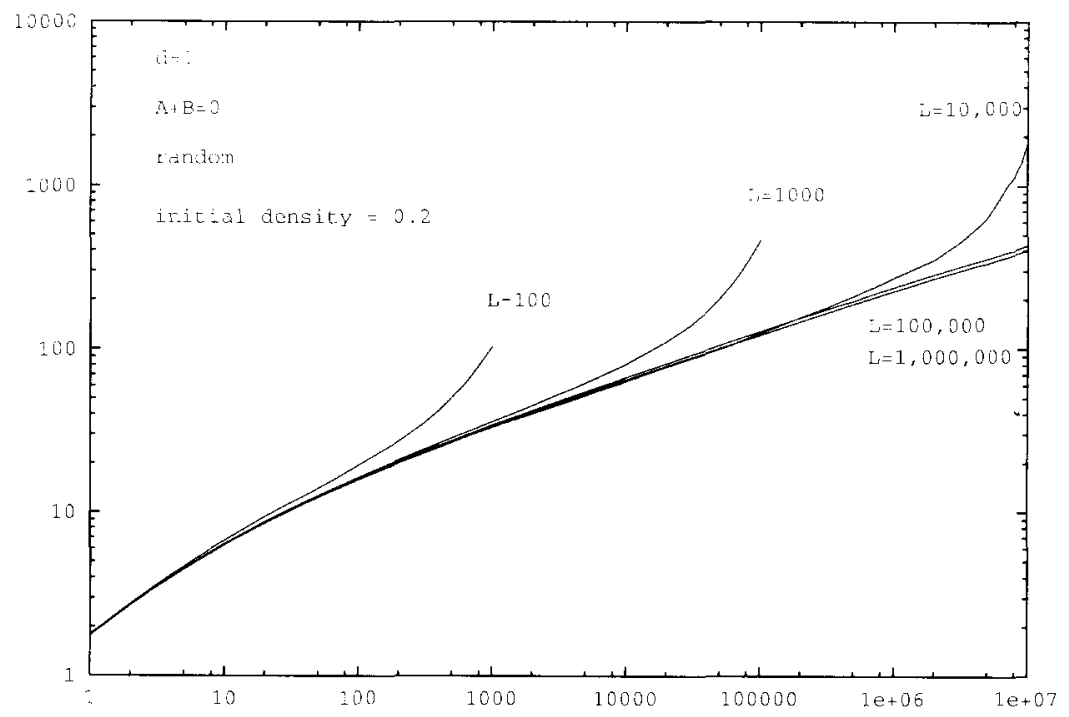

Fig. 1. $\langle\rho\rangle^{-1}-\rho_{0}^{-1}$ versus $t$ for lattices of various sizes $L$ in one dimension with a random initial condition. The initial density of each species in number per lattice site is $\rho_{0}=0.2$ in all cases.

until the asymptotic behavior is reached. We characterize the various regimes and estimate the crossover times. We present numerical simulation results and analyze them in terms of our model. Only qualitative results are presented here with some characteristic examples, while a detailed analysis will be given in [9].

The summary of our results is given in Fig. 1, which is a plot of $\langle\rho\rangle^{-1}-\rho_{0}^{-1}$ versus $t$ for one dimensional lattices of various sizes, and is the result of Monte Carlo simulation data. We observe the various regimes in this figure as follows: First comes a classical regime at $t=0$. The rate equation here is the classical rate equation: $\left.\dot{\rho}_{\mathrm{g}}(t)\right|_{t=0}=$ $-k \rho_{\mathrm{g}}^{2}(0)$, where $\rho_{\mathrm{g}}(t)$ is the average global density, and $\rho_{\mathrm{g}}(0)=\langle\rho(\boldsymbol{r}, 0)\rangle \equiv \rho_{0}$. Thus, very near $t=0$ the solution is $\rho_{\mathrm{g}}(t)=\rho_{0} /\left(1+k \rho_{0} t\right)$ and also $\langle\rho(\boldsymbol{r}$, $t)\rangle=\rho_{0} /\left(1+k \rho_{0} t\right)$. The initial slope at very early times is the same as that of the A $+\mathrm{A}$ problem, which is the classically expected value of 1 .

Then comes an intermediate regime where we have $\langle\rho(\boldsymbol{r}, t)\rangle \sim t^{-d / 2}$ for $d \leqslant 2$, and $\langle\rho(\boldsymbol{r}, t)\rangle \sim t^{-1}$ for $d>2$. This behavior is nonclassical in one dimension, and is completely analogous to the nonclassical regime of the reactions $A+A \rightarrow 0$ and $A+A \rightarrow A$. In those cases the slower-than-classical decay $t^{-1 / 2}$ has been well documented to arise from the "depletion zone" that forms around each surviving reactant after the initially very close pairs have quickly reacted [1]. Diffusion in one dimension is known to be an ineffective mechanism toward refilling the depletion zone. The same happens here in the $A+B \rightarrow 0$ reaction: After the rapid initial reaction of very nearby $A-B$ pairs (classical regime) each surviving $A$ and $B$ is surrounded by a region relatively poor in the other species [10]. These depletion zones are not efficiently repopulated by diffusion. In Fig. 1 we see that the slope in this region is about 0.55 (over the first 10 steps).

After a sufficiently long time has elapsed, it is well known [1-8] that the system evolves towards macroscopic segregation and that $\langle\rho(\boldsymbol{r}, t)\rangle \sim t^{-d / 4}$ for $d<4$. At very long times the largest lattices show the Zeldovich behavior. For the largest lattice we find an average slope of 0.249 over the last decade of the simulation. The crossover time from the $\mathrm{A}+\mathrm{A}$ depletion zone behavior to the Zeldovich behavior is discussed below and found to be $t_{\mathrm{s}} \sim 10^{2}$.

Finally, at long times finite volume effects will affect the results of computer simulations. It is well 
known that this causes an exponential decay of the density, $\langle\rho(\boldsymbol{r}, t)\rangle \sim \mathrm{e}^{-\chi t}$, where $\chi$ is a constant inversely proportional to the size of the system. This behavior is expected to take over when the size of each aggregate is of the order of the size of the system, or, if the initial density is too small to lead to segregation before finite size effects set in, when the average distance between particles is of the order of the size of the system. This is also shown clearly in Fig. 1, where the smaller lattices show the "peel off" due to finite size effects.

The "major" crossover time $t_{\mathrm{s}}$ and average crossover density $\rho_{\mathrm{s}}$ for $d \leqslant 4$ occur when the $t^{-d / 4}$ segregation behavior sets in. This occurs from the depletion zone regime. We write the crossover density as a fraction of the initial density: $\rho_{\mathrm{s}} \sim f_{\mathrm{d}} \rho_{0}$, where $f_{\mathrm{d}}$ is a dimension-dependent fraction assumed to be of $O(1)$ and independent of the initial density. This crossover time is found to be [9]:

$t_{\mathrm{s}} \sim \frac{1}{8 \pi D \rho_{0}^{2 / d} f_{\mathrm{d}}^{4 / d}}$

The equations for $\rho_{\mathrm{s}}$ and $t_{\mathrm{s}}$ can be verified with the numerical data from Fig. 1 . The value of $f_{\mathrm{d}}$ must be determined from the simulations (but need only be determined for one value of $\rho_{0}$ ). If our assumption that $f_{\mathrm{d}}$ is independent of $\rho_{0}$ is correct, then $t_{\mathrm{s}} \rho_{0}^{2 / d}$ should be independent of the initial density $\rho_{0}$. Likewise, the ratio of the crossover density to the initial density, $\rho_{\mathrm{s}} / \rho_{0}$, should be independent of $\rho_{0}$. Our simulations confirm these behaviors.

If the initial density is high enough for there to be a segregation regime, finite size effects set in when the aggregates of like molecules are of the order of the size of the system. It is known [10] that the linear size of the aggregates on the average grows with time as $a_{\mathrm{d}}(D t)^{1 / 2}$. Here $a_{\mathrm{d}}$ is a dimensiondependent constant of proportionality, which appears [10] to be approximately 4 in one dimension. Finite size effects are thus expected to set in at a time $t_{\mathrm{f}}$ such that the average linear size of the aggregates in some fraction $g_{\mathrm{d}} \sim \mathrm{O}(1)$ of the linear size $L$ of the system, $a_{\mathrm{d}}\left(D t_{\mathrm{f}}\right)^{1 / 2} \sim g_{\mathrm{d}} L$, i.e.,

$t_{\mathrm{f}} \sim g_{\mathrm{d}}^{2} \frac{L^{2}}{a_{\mathrm{d}}^{2} D}$.
The dimension-dependent fraction $g_{\mathrm{d}}$ is expected to be independent of the initial density, and must be determined from simulations. For $L=100$ we find that finite size effects begin to set in when $t=$ $t_{\mathrm{f}} \sim 20$. According to Eq. (2), the average size of the aggregates is approximately $g_{1} L \sim 4\left(D t_{\mathrm{f}}\right)^{1 / 2} \sim 13$, i.e., $g_{1}=0.13$. Thus, each aggregate of each species is on the average larger than $1 / 10$ of the size of the system (which of course must contain at least two aggregates). Since we assume that the fraction $g_{\mathrm{d}}$ is independent of $L$, we can use the value obtained above, $g_{1} \sim 0.13$, to predict the crossover times $t_{\mathrm{f}}$ for the other lattices. We find for $L=1000$ that $t_{\mathrm{f}} \sim 210^{3}, \quad$ and for $L=10000$ we obtain $t_{\mathrm{f}} \sim 210^{5} . \quad$ Both are consistent with the simulation results of Fig. 1.

We conclude that the following sequence of behaviors is present: First at early times the $\mathrm{A}+\mathrm{B}$ reaction behaves the same way as the $A+A$ reaction. Both exhibit classical behavior at very early times but quickly cross over to the depletion zone behavior $t^{-d / 2}$ for $d \leqslant 2$. This parallel behavior continues up to a time $t_{\mathrm{s}}$, when segregation effects begin in the $\mathrm{A}+\mathrm{B}$ reaction. We estimate the time $t_{\mathrm{s}}$ in Eq. (1) and, in particular, predict the product $t_{\mathrm{s}} \rho_{0}^{2 / d}$ to be independent of the initial density $\rho_{0}$. The transition to the Zeldovich regime is therefore expected at shorter times for higher initial densities. We also predict the crossover density $\rho_{\mathrm{s}}$ to be proportional to $\rho_{0}$. Our simulations confirm these predictions quantitatively. Thus, a simulation for a single initial density in a particular dimension allows us to predict this crossover quantitatively for any other initial density. We find that in one dimension $t_{\mathrm{s}} \rho_{0} \sim \mathrm{O}\left(10^{0}\right)-\mathrm{O}\left(10^{1}\right)$ and $\rho_{\mathrm{s}} / \rho_{0} \sim$ $0.3-0.5$. From these results we can also predict whether the transition to the Zeldovich regime occurs earlier or later in two dimensions than in one dimension for a given value of $\rho_{0}$. We find that $t_{\mathbf{s}}(2$ dimensions $) / t_{\mathrm{s}}(1$ dimension $)=K \rho_{0}$, where $K$ is of $\mathrm{O}\left(10^{1}\right)-\mathrm{O}\left(10^{2}\right)$. Thus, except for very low initial densities, the transition will set in later in two dimensions.

The Zeldovich regime in all cases ends when finite lattice size effects set in. These effects begin when the average linear size of each kind of aggregate (which grows in time as $t^{1 / 2}$ ) is within an order of magnitude of the size of the system, independ- 
ently of the size of the system. In other words, the finite size effects for lattices of linear size $L$ start at a time $t_{\mathrm{f}} \sim L^{2} \quad[\mathrm{cf}$. Eq. (2)]. Again, we have determined dimension-specific parameters for this crossover from our simulations. Furthermore, from the finite size results we obtain information about the segregation process in infinite lattices.

Finally, we stress that our simulations are in excellent agreement with our theoretical predictions.

\section{Acknowledgements}

We gratefully acknowledge support from the U.S. DOE grant No. DE-FG03-86ER13606 (KL), from the NSF grant No. DMR-9111622 (RK), and from NATO grant No. CRG 920029 (PA).

\section{References}

[1] R. Kopelman, J. Stat. Phys. 42 (1986) 185; Science 241 (1988) 1620

[2] V. Kuzovkov and E. Kotomin, Rep. Prog. Phys. 51 (1988) 1479.

[3] A.A. Ovchinnikov and Y.G. Zeldovich, Chem. Phys. 28 (1978) 215.

[4] D. Toussaint and F. Wilczek, J. Chem. Phys. 78 (1983) 2642.

[5] K. Kang and S. Redner, Phys. Rev. Lett. 52 (1984) 955.

[6] M. Bramson and J.L. Lebowitz, J. Stat. Phys. 62 (1991) 297.

[7] L.W. Anacker and R. Kopelman, Phys. Rev. Lett. 58 (1987) 289.

[8] D.C. Torney and H.M. McConnell, J. Phys. Chem. 87 (1983) 1441; Proc. Roy. Soc. London, Ser. A, 387 (1983) 147.

[9] P. Argyrakis, R. Kopelman and K. Lindenberg, Chem. Phys., to appear.

[10] F. Leyvraz and S. Redner, Phys. Rev. Lett. 66 (1991) 2168; Phys. Rev. A 46 (1992) 3132. 